\title{
Suppression of Ripe Rot on 'Zesy002' Kiwifruit with Commercial Agrochemicals
}

\author{
Yong Ho Shin ${ }^{1}$, Magda Ledesma ${ }^{1}$, Sonia Whitman ${ }^{2}$, Joy Tyson ${ }^{3}$, Birgit Zange ${ }^{4}$, Ki Deok Kim ${ }^{5}$, and \\ Yong Chull Jeun (1) ${ }^{1,6 *}$ \\ ${ }^{1}$ Sustainable Agriculture Research Institute, Jeju National University, Jeju 63243, Korea \\ ${ }^{2} Z$ Zespri International Limited, Mt Maunganui 3149, New Zealand \\ ${ }^{3}$ The New Zealand Institute for Plant \& Food Research Limited (PFR), Auckland 1142, New Zealand \\ ${ }^{4}$ Faculty of Horticulture and Food Technology, University of Applied Sciences, Weihenstephan-Triesdorf, Freising \\ 85354, Germany \\ ${ }^{5}$ Department of Plant Biotechnology, Korea University, Seoul 02841, Korea \\ ${ }^{6}$ The Research Institute for Subtropical Agriculture and Biotechnology, Jeju National University, Jeju 63604, Korea
}

(Received on March 12, 2021; Revised on June 2, 2021; Accepted on June 2, 2021)

Ripe rot caused by Botryosphaeria dothidea is one of the serious diseases of postharvest kiwifruit. In order to control ripe rot on Actinidia chinensis cultivar 'Zesy002', several commercial agrofungicides were selected by an antifungal test on an artificial medium. Furthermore, disease suppression by the selected fungicides was evaluated on the kiwifruit by inoculation with a conidial suspension of $B$. dothidea. On the artificial media containing boscalid + fludioxonil was shown to be the most effective antifungal activity. However, in the bio-test pyraclostrobin + boscalid and iminoctadinetris were the most effective agrochemicals on the fruit. On the other hand, the infection structures of $B$. dothidea on kiwifruit treated with pyraclostrobin + boscalid were observed with a fluorescent microscope. Most of the fungal conidia had not germinated on the kiwifruit treated with the agrochemicals whereas on the un-

\section{*Corresponding author.}

Phone) +82-64-754-3319, FAX) +82-64-725-2351

E-mail) ycjeun@jejunu.ac.kr

ORCID

Yong Chull Jeun

https://orcid.org/0000-0002-9084-1393

Handling Editor : Ki Woo Kim

(c) This is an Open Access article distributed under the terms of the Creative Commons Attribution Non-Commercial License (http:// creativecommons.org/licenses/by-nc/4.0) which permits unrestricted noncommercial use, distribution, and reproduction in any medium, provided the original work is properly cited.

Articles can be freely viewed online at www.ppjonline.org. treated fruit the fungal conidia had mostly germinated. Electron microscopy of the fine structures showed morphological changes to the conidia and branch of hyphae on the kiwifruit pre-treated with pyraclostrobin + boscalid, indicating its suppression effect on fungal growth. Based on this observation, it is suggested that ripe rot by $B$. dothidea may be suppressed through the inhibition of conidial germination on the kiwifruit treated with the agrochemicals.

Keywords : dimple rot, fluorescence microscopy, infection structure, plant protection, postharvest disease

Kiwifruit contains a lot of vitamin $\mathrm{C}$ and low calories and gives beneficial healthy effects to human's body. Furthermore, it has been reported that kiwifruit had effects to prevent cardiovascular disease, cancer, and other degenerative disorders (Singletary, 2012). For these reasons kiwifruit is one of the popular fruit in South Korea and extent of kiwifruit cultivation was taken at 1,300 ha in 2015 (Kim et al., 2017a). The consumption of kiwifruit in South Korea is increasing and reached approximately 35,000 tons in 2018 less than 10,000 tons were harvested in Korea (Tridge, 2021). However, the amount of kiwifruit yield could not be increased as much as expected because of outbreak of the diseases.

One of the most serious diseases in kiwifruit vines is bacterial canker caused by Pseudomonas syringae pv. actinidiae (Psa; Greer and Saunders, 2012). In many South Korean 
kiwifruit orchards, the susceptible kiwifruit cultivar 'Hort16A' has been replaced with another cultivar, 'Zesy002', which is tolerant to Psa (Donati et al., 2014). However, over the past decade postharvest diseases have occurred on 'Zesy002' kiwifruit which was found in several orchards of Jeju Island.

During the storage of kiwifruit several diseases often occur during low temperature storage. In a study of postharvest rots of 'Hort16A' in New Zealand most of the rots were found to be caused by Diaporthe spp., Botrytis cinerea, or Botryosphaeria dothidea (Manning et al., 2003). A survey of Korean kiwifruit orchards in 1999 showed that as much as $32 \%$ of kiwifruit had soft rot caused by these diseases (Koh et al., 2003). Among these, B. dothidea was the main fungus causing postharvest diseases and accounted for $83.3 \%$ of the fungal pathogens (Koh et al., 2003).

In Korea, 20.5\% of kiwifruit cultivar 'Hayward' (Actinidia chinensis var. deliciosa) was lost with ripe rot caused by $B$. dothidea in 2014 (Kim and Koh, 2018). Another cultivar of $A$. chinensis var. deliciosa (Gamrok) was known to be more susceptible to $B$. dothidea because of its higher sugar contents than other cultivars (Kim et al., 2017b). To control postharvest rot on kiwifruit, many agrochemicals were tested in kiwifruit orchards, especially cultivating kiwifruit cultivar A. chinensis 'Zesy002'.

In this study, in order to evaluate the relative efficacy of the currently used chemical products, the direct protection rate of the chemicals were evaluated by agar plate assays. To further optimize agrochemicals used on-orchard, agrochemicals which were shown to inhibit antifungal activity in the agar plate test were assayed for efficacy in a bio-test with 'Zesy002' fruit. Furthermore, to reveal the disease suppression mechanism on kiwifruit by these chemicals, the infection structures of $B$. dothidea were observed using a fluorescence microscope.

\section{Material and Methods}

Fruit material. Kiwifruit (Actinidia chinensis var. chinensis cultivar 'Zesy002') were supplied by Zespri Co. Ltd. in Jeju Korea. The kiwifruit were sterilized with $1 \%$ sodium hypochlorite, $70 \%$ ethyl alcohol each for $60 \mathrm{~s}$ and washed with sterilized water three times. After drying at room temperature the kiwifruit were used for inoculation.

Fungal inoculum. B. dothidea isolate $\mathrm{C} 1.1$ was isolated from 'Zesy002' kiwifruit showing ripe rot symptom, which is visually similar to dimple rot, from an orchard of Jeju Island Korea during the 2018 harvest season (Ledesma et al., 2021). This isolate was held in Jeju University plant pa- thology general culture collection at $-80^{\circ} \mathrm{C}$ and used in this experiment. For sporulation, $B$. dothidea was grown on an oatmeal agar medium (Becton, Dickinson and Company, Claix, France) under aerobic conditions at $28^{\circ} \mathrm{C}$ with 7,000 lux in daytime for 7 days. Mycelium of $B$. dothidea was scraped with a loop and incubated another 7 days under the same conditions. In the plate $10 \mathrm{ml}$ of sterilized water was added and conidia were harvested with a brush. The conidial suspension was filtered with double folded Miracloth (Calbiochem, Darmstadt, Germany). The concentration of inoculum was adjusted to $1 \times 10^{5}$ conidia $/ \mathrm{ml}$ using a haemocytometer (Hausser Scientific Inc., Horsham, PA, USA) and used as inoculum.

\section{Agrochemical evaluation}

In vitro test. The effect of several agrochemicals were tested in potato dextrose agar (PDA; Becton, Dickinson and Company) medium. All chemicals were added into the PDA at the recommended concentration for field application, respectively. Some of the agrochemicals were subsequently diluted because the inoculated fungi did not grow. The list of agrochemicals and recommended concentrations are given in Table 1. As the control, PDA without chemicals was used.

Mycelium of $B$. dothidea $(\varnothing=5 \mathrm{~mm})$ was inoculated on the PDA medium added with the different agrochemicals and incubated at $25^{\circ} \mathrm{C}$ for 7 days. The diameter of the colony was measured with a ruler. This experiment was replicated three times including three PDA plates each.

In vivo test. Agrochemicals which revealed direct anti-

Table 1. Agrochemicals used and their applied concentration in potato dextrose agar media

\begin{tabular}{lcc}
\hline Ingredients & $\begin{array}{c}\text { Concentration } \\
(\mathrm{ppm})\end{array}$ & $\begin{array}{c}\text { Diluted } \\
\text { concentration } \\
(\mathrm{ppm})\end{array}$ \\
\hline Iminoctadine-tris & 1,000 & $-^{\mathrm{a}}$ \\
Azoxystrobin & 1,000 & - \\
Tebuconazole & 500 & 50 \\
Thiophanate methyl & 1,000 & - \\
Iprodione & 1,000 & 100 \\
Boscalid & 500 & - \\
Trifloxystrobin & 250 & 10 \\
Boscalid-fludioxonil & 1,000 & - \\
Fluazinam & 500 & - \\
Fluopyram & 250 & - \\
Cyprodinil & 500 & - \\
Pyraclostrobin + boscalid & 500 & \\
\hline
\end{tabular}

${ }^{\mathrm{a}}$ Not tested. 
fungal effect on agar plates such as iprodione, tebuconazole, boscalid-fludioxonil, fluazinam, iminoctadinetris, thiophanate methyl, trifloxystrobin, cyprodinil and pyraclostrobin + boscalid were used in this experiment. All chemicals were applied to 'Zesy002' kiwifruit previously wounded with a sterile stick $(\varnothing=0.5 \mathrm{~mm})$ at the recommended concentration for field application. After drying at room temperature the treated fruit were used for fungal inoculation.

A drop of $70 \mu \mathrm{l}$ of conidial suspension of $B$. dothidea with $0.01 \%$ Tween-20 was inoculated onto the wounded part of each kiwifruit. The inoculated fruit were placed in an incubator maintained at $28^{\circ} \mathrm{C}, 90 \%$ relative humidity, for 7 days. As a control, sterilized $0.01 \%$ Tween- 20 solution was applied instead of the conidial suspension. The symptoms on the fruit were examined and the lesions were measured with a ruler. The experiments were separately replicated 3 times which contained six kiwifruit each treatment.

Observation with a fluorescence microscope. To investigate the infection behavior of $B$. dothidea kiwifruit treated with pyraclostrobin + boscalid was observed with a fluorescence microscope (BX60, Olympus, Tokyo, Japan). The kiwifruit surfaces were inoculated with $60 \mu \mathrm{l}$ of $B$. dothidea conidial suspension and the inoculated parts were excised with a razor in size of $5 \times 5 \mathrm{~mm}^{2}$ at 3,6 , and $12 \mathrm{~h}$ after inoculation. The peel tissues were fixed with $2 \%$ glutaraldehyde in sodium phosphate buffer $(\mathrm{pH} 7.2)$ at $4^{\circ} \mathrm{C}$ for $2 \mathrm{~h}$. After fixation, the samples were washed with sodium phosphate buffer three times (10 min each) and dyed with $0.2 \%$ diethanol (UVtex-2B, Polysciences, Inc., Muellheim, Germany) for $30 \mathrm{~m}$ at room temperature. After washing with $\mathrm{H}_{2} \mathrm{O}$ three times the samples were mounted on glass slides with 70\% glycerin (glycerin, OCI Company Ltd., Seoul, Korea). Infected sites were observed with a fluorescent filter set (exciter filter, BP 400-440; interference beam splitter, FT 460; barrier filter, LP 470). The germinated conidia were counted and the length of hyphae was measured. Experiments were separately replicated three times using three samples for each treatment.

Scanning electron microscopy of the kiwifruit after fungal inoculation. Kiwifruit surfaces of 'Zesy002' pre-treated with agrichemicals and then inoculated with $B$. dothidea were observed at 1,2 , and 3 days after inoculation using a scanning electron microscope (FE-SEM Mira3, Tescan Ltd., Brno, Czech Republic).

The inoculated kiwifruit were cut to $0.4 \times 0.6 \mathrm{~mm}^{2}$ using a sterile blade. Fixation, dehydration and embedding of the fruit were performed according to Hayat (1989). The leaf samples were fixed in $2 \%(\mathrm{v} / \mathrm{v})$ glutaraldehyde in 0.05 $\mathrm{M}$ phosphate buffer (pH 7.4) for $2 \mathrm{~h}$. After washing with phosphate buffer for $10 \mathrm{~min}$ three times each, post fixation was performed in $2 \%(\mathrm{v} / \mathrm{v})$ osmium tetroxide in phosphate buffer for $2 \mathrm{~h}$ at room temperature. After washing three times, the samples were dehydrated through an alcohol series $(30 \%, 50 \%, 70 \%, 90 \%$, and $100 \%$ two times for 30 min each). The samples were gently dried using a critical point drier (EM CPD 300, Leica, Jena, Germany). Samples were mounted on metallic stubs, gold-coated $(\sim 100 \AA)$ with a sputter coater (Q150R Plus - Rotary Pumped Coater, Quorum Technologies Ltd., Sussex, UK) and viewed under the FE-SEM at $20 \mathrm{kV}$.

Statistical analysis. Data of diameters of mycelia on the PDA for determining the efficacy of agrochemicals, disease severity on kiwifruit treated with agrochemicals, rate of conidial germination, and hyphal lengths on surfaces of kiwifruit were analyzed with Duncan's multiple range test using statistical analysis system (SAS) program version 9.0 (SAS Institute Inc., Cary, NC, USA). Statistical significance was considered at $P<0.05$.

\section{Results}

Antifungal effect on PDA medium amended with agrochemicals. Hyphal growth suppression of $B$. dothidea by 12 commercial agrochemicals was evaluated on PDA medium. At 7 days after inoculation, hyphal growth was inhibited on all of the agrochemical amended media compared with the untreated control (Fig. 1). Especially, mycelium of $B$. dothidea did not grown on PDA amended with tebuconazole, iprodione and boscalid + fludioxonil at field application concentration (data not shown). Even when tebuconazole or iprodione were diluted 10 times, the antifungal effect was high compared with other agrochemicals tested (Fig. 2). Above all, PDA treated with boscalid + fludioxonil diluted 100 times was shown to have the best suppression effect compared with other chemicals in the in vitro experiment (Fig. 2).

Disease suppression on kiwifruit treated with agrochemicals. To test the disease suppression effect on kiwifruit treated with the agrochemicals, $B$. dothidea was inoculated onto kiwifruit pre-treated with the most effective nine agrochemicals selected from the in vitro experiment. On untreated kiwifruit, symptoms of $B$. dothidea were visible at 3 days after inoculation. The lesion sizes were larger on the untreated fruit compared with all of the chemicals- 


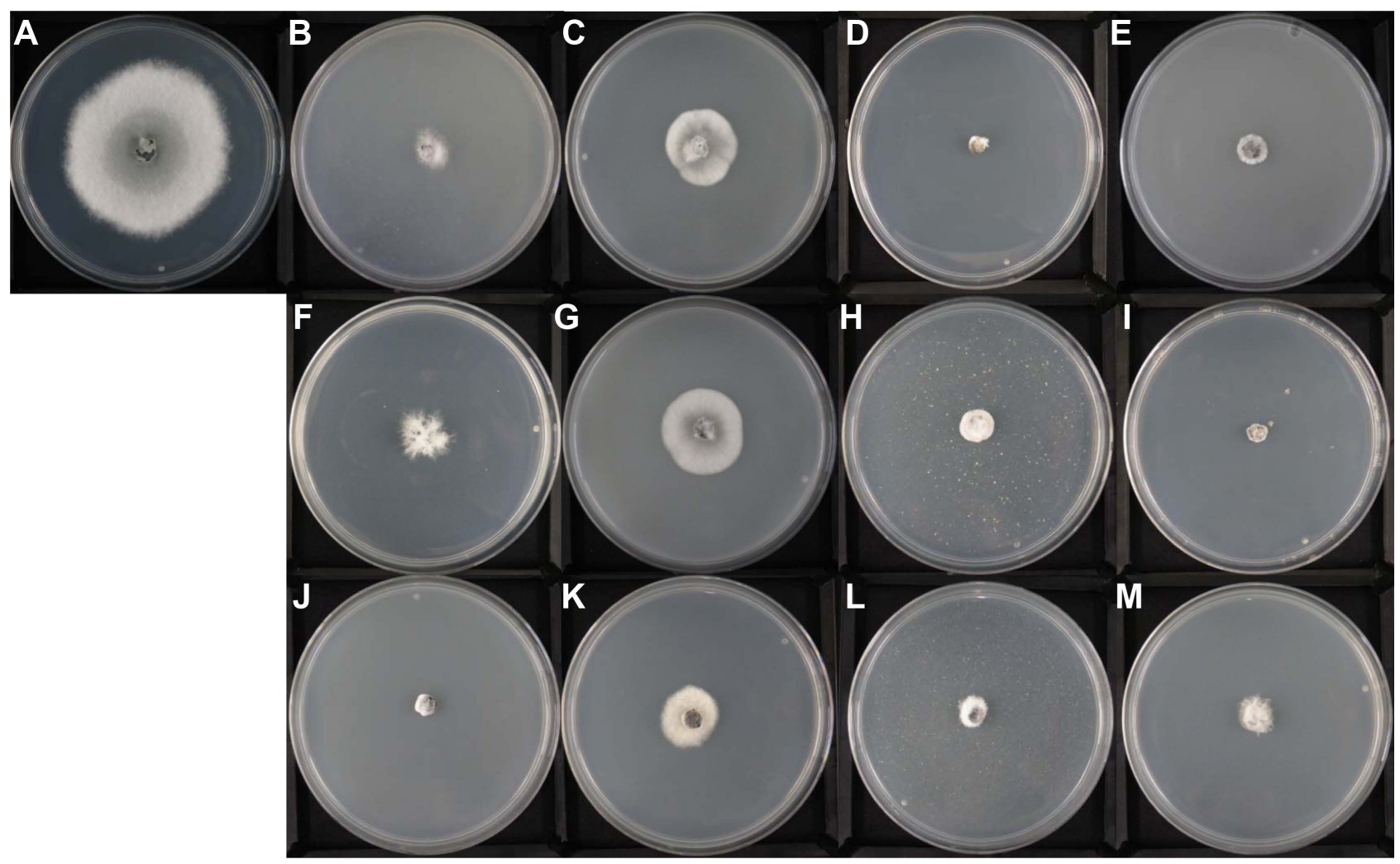

Fig. 1. Mycelium of Botryosphaeria dothidea grown on potato dextrose agar (PDA) medium added with different agrichemicals. The present photos were taken at 7 days after inoculation with the fungus. The recommended concentration for field application were added on the PDA medium, respectively. (A) Control. (B) Iminoctadine-tris. (C) Azoxystrobin. (D) Tebuconazole dilluted 10 times. (E) Fluopyram + thiophanate-methyl. (F) Iprodione dilluted 10 times. (G) Boscalid. (H) Trifloxystrobin. (I) Boscalid + fludioxonil dilluted 100 times. (J) Fluazinam. (K) Fluopyram. (L) Cyprodinil. (M) Pyraclostrobin + boscalid.

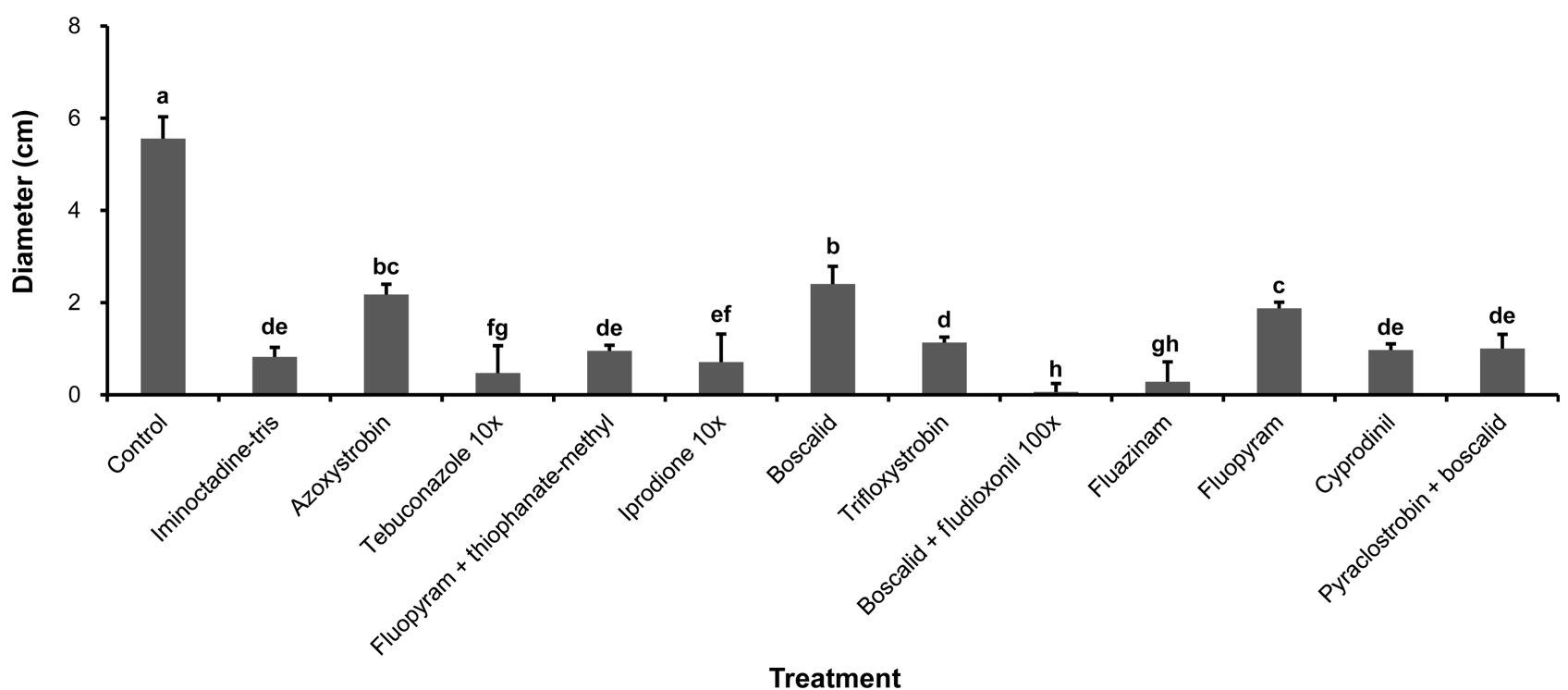

Fig. 2. Diameter of mycelium of Botryosphaeria dothidea grown on potato dextrose agar (PDA) medium added with different agrichemicals. The present photos were taken at 7 days after inoculation with the fungus. The recommended concentration for field application and were added on the PDA medium, respectively. Different letters on the columns indicate significant differences $(P<0.05)$ according to Duncan's multiple test. 

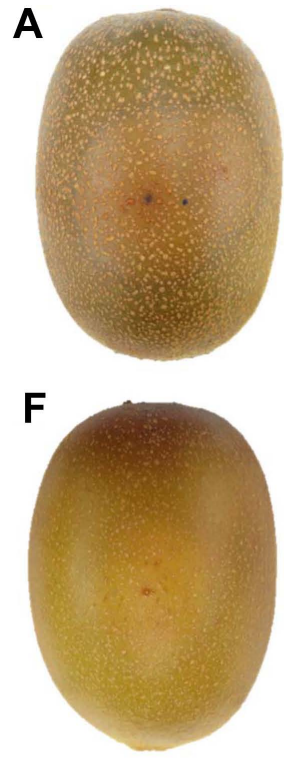

B

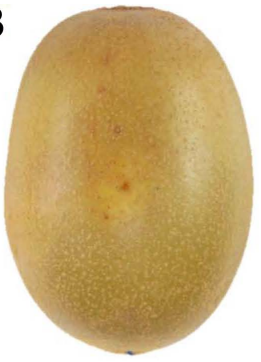

G

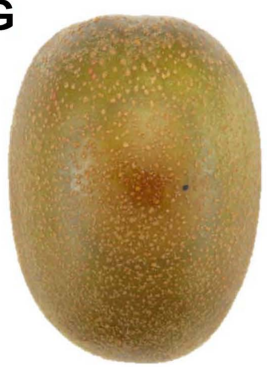

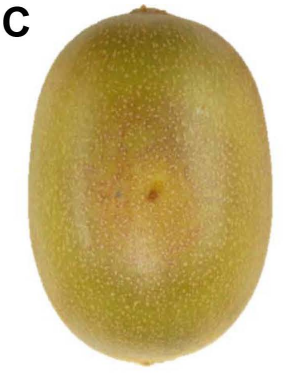

H

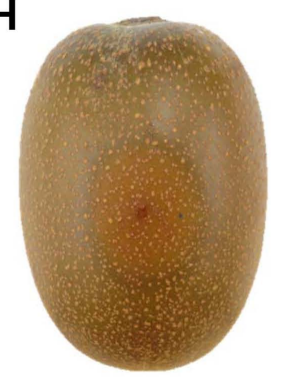

D

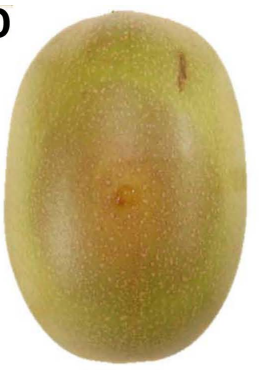

I

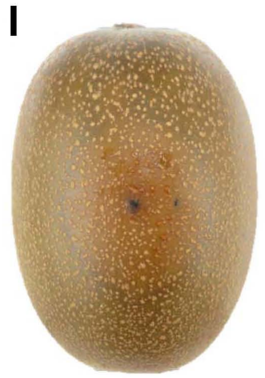

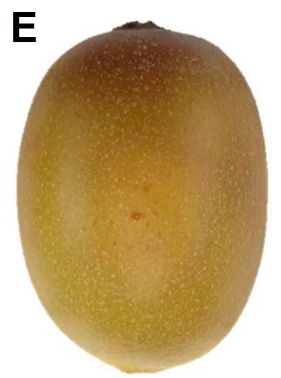

J

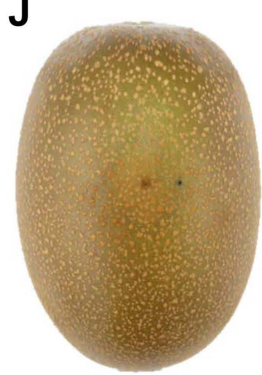

Fig. 3. Wounded 'Zesy002' kiwifruit control (A), pre-treated with iminoctadine-tris (B), tebuconazole (C), iprodione (D), boscalid + fludioxonil (E), fluazinam (F), fluopyram + thiophanate-methyl (G), trifloxystrobin $(\mathrm{H})$, cyprodinil (I), and pyraclostrobin + boscalid (J) which were inoculated with conidial suspension of Botryosphaeria dothidea and as a negative control both untreated/uninoculated kiwifruit $(\mathrm{F})$. The recommended concentration for field application were pre-treated before fungal inoculation, respectively. The concentration of the conidial suspension of $B$. dothidea were $1 \times 10^{5}$ conidia/ml. The present photos were taken at 7 days after inoculation with the fungus.

treated kiwifruit.

At 7 days after inoculation, the symptom diameter on all of the agrochemical pre-treated kiwifruit were reduced compared with those symptoms on the untreated control
(Fig. 3). Especially, pyraclostrobin+boscalid and iminoctadine-tris were the most effective agrochemicals on kiwifruit, on which the disease suppression rates were $75 \%$ and $44 \%$ compared with the untreated control, respectively (Fig.

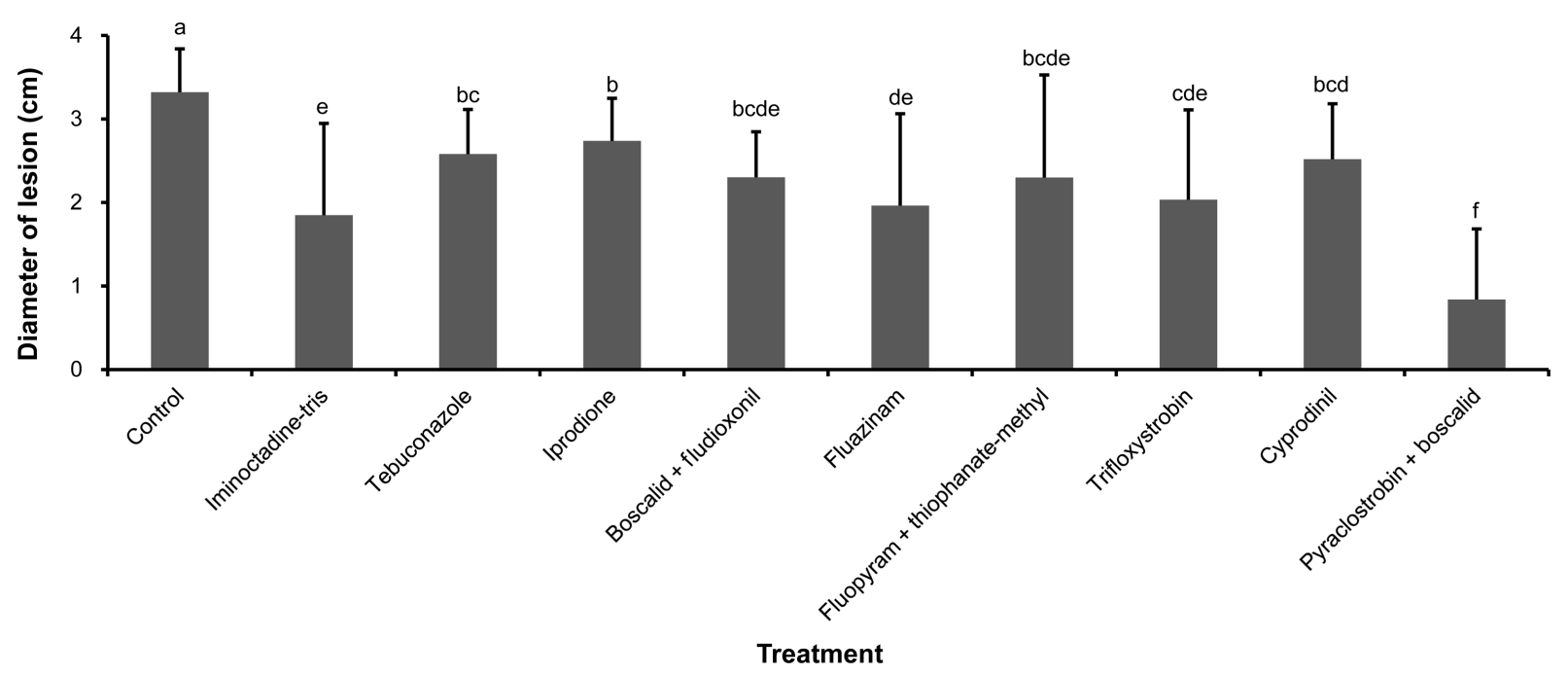

Fig. 4. Diameter of ripe rot lesion on wounded 'Zesy002' kiwi fruits treated with agrichemicals after inoculated with conidial suspension of Botryosphaeria dothidea. The recommended concentration for field application were pre-treated before fungal inoculation, respectively. The concentration of the conidial suspension of $B$. dothidea were $1 \times 10^{5}$ conidia $/ \mathrm{ml}$. The diameter of lesions were measured at 7 days after inoculation with the fungus. Different letters on the columns indicate significant differences $(P<0.05)$ according to Duncan's multiple test. 
Table 2. Germination rate of conidia of Botryosphaeria dothidea on the surface of kiwifruit 'Zesy002' untreated and treated with agrochemical pyraclostrobin + boscalid

\begin{tabular}{|c|c|c|c|c|}
\hline \multirow{3}{*}{ Treatment } & \multicolumn{4}{|c|}{ Germination rate $(\%)$} \\
\hline & \multicolumn{3}{|c|}{ Hours after inoculation (h) } & \multirow{2}{*}{ DMRT } \\
\hline & 3 & 6 & 12 & \\
\hline Untreated & $13.7 \pm 19.1^{b}$ & $72.5 \pm 15.4$ & $96.9 \pm 2.1$ & $\mathrm{a}$ \\
\hline Pyraclostrobin + boscalid & $0.0 \pm 0.0$ & $0.1 \pm 0.3$ & $0.0 \pm 0.0$ & $\mathrm{~b}$ \\
\hline
\end{tabular}

aThe letter indicates significant differences according to Duncan's multiple range test (DMRT) $(P<0.01)$.

${ }^{\mathrm{b}}$ Mean \pm standard deviation.

4). This indicates that the agrochemicals have a disease suppression effect on kiwifruit.

Fluorescent microscopy. To reveal the mechanism of disease suppression on kiwifruit treated with pyraclostrobin + boscalid, which was shown to be the most effective against $B$. dothidea, the infection structure of $B$. dothidea on kiwifruit were observed with a fluorescence microscope. At 3 $\mathrm{h}$ after inoculation some conidia were germinated on the untreated kiwifruit and $6 \mathrm{~h}$ later most conidia were germinated (Table 2). Hyphal growth developed rapidly and the surfaces of kiwifruit were covered with hyphae $12 \mathrm{~h}$ after inoculation (Fig. 5A-C).

However, most conidia did not germinate on the agrochemical-treated kiwifruit during the whole observation period from 3 to $12 \mathrm{~h}$ after inoculation (Table 2, Fig. 5DF). These observations indicated that the agrochemicals inhibited the conidial germination which might result in the suppression of disease severity. Even on kiwifruit treated with pyraclostrobin + boscalid 100 times diluted from field rates, most conidia had not germinated at $12 \mathrm{~h}$ after inoculation (data not shown), indicating that pyraclostrobin + boscalid was the most effective fungicide to suppress ripe rot on kiwifruit.

Scanning electron microscopy. On the untreated kiwifruit, conidia of $B$. dothidea were morphologically intact and mostly germinated at 1 day after inoculation (Fig. 6A). One day later the hyphae of the fungus had grown on the surface of the kiwifruit and were extended widely at 3 days after inoculation (Fig. 6B and C).

However, on the pyraclostrobin + boscalid pre-treated kiwifruit, some conidia of $B$. dothidea were morphologically changed (Fig. 6D, arrow) and most conidia had not germinated. The tapered conidia were often found at 2 days after inoculation (Fig. 6E, arrow) and most conidia still had not
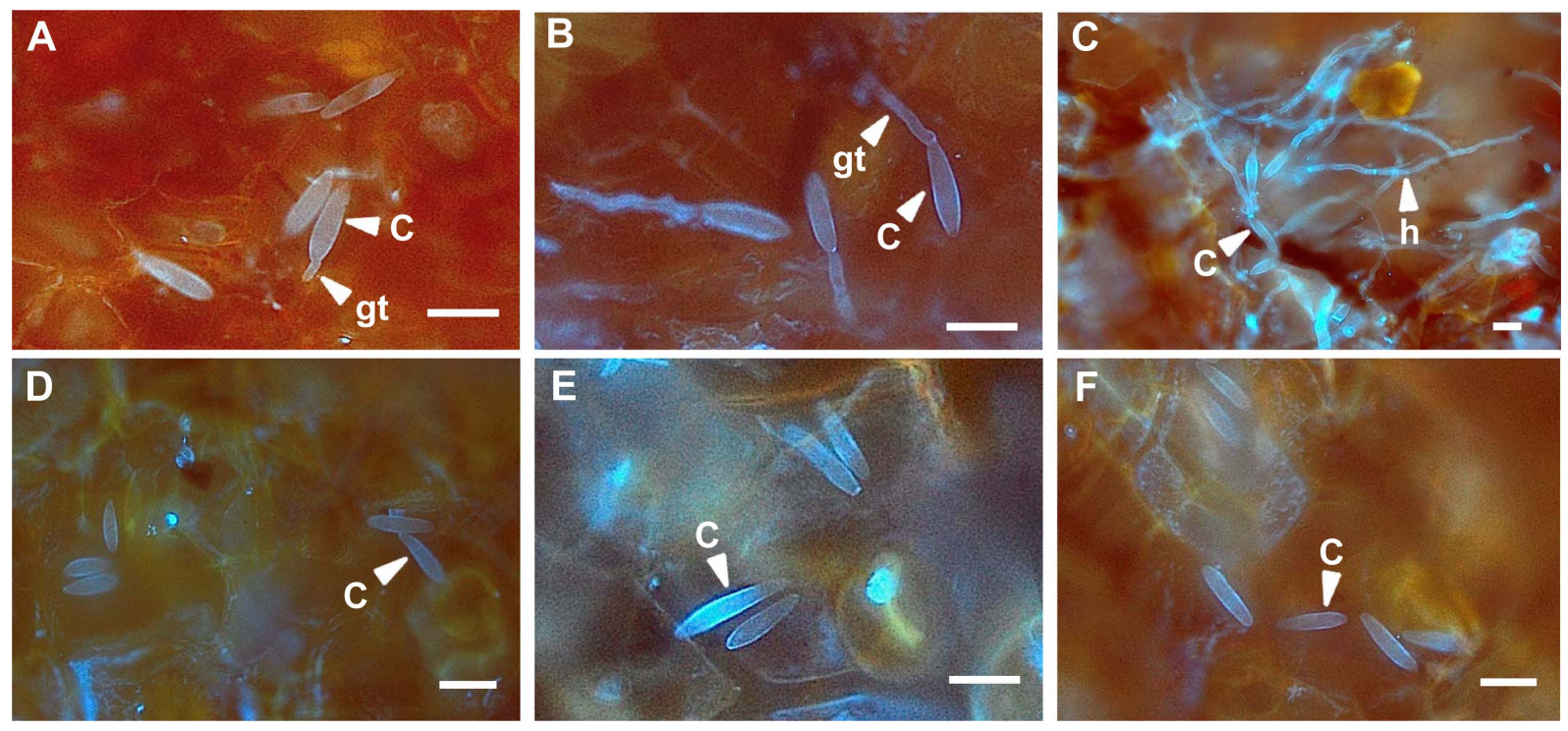

Fig. 5. Fluorescent microscopic observation of infection structures at 3, 6, 12 hours after inoculation on peels of kiwifruits untreated (AC) and fungicide pyraclostrobin-boscalid (D-F). The concentration of fungicide and conidia suspension of Botryosphaeria dothidea were field application and $1 \times 10^{5}$ conidia/ml. Scale bars $=20 \mu \mathrm{m}$. c, conidium; $\mathrm{h}$, hyphae; gt, gum tube. 

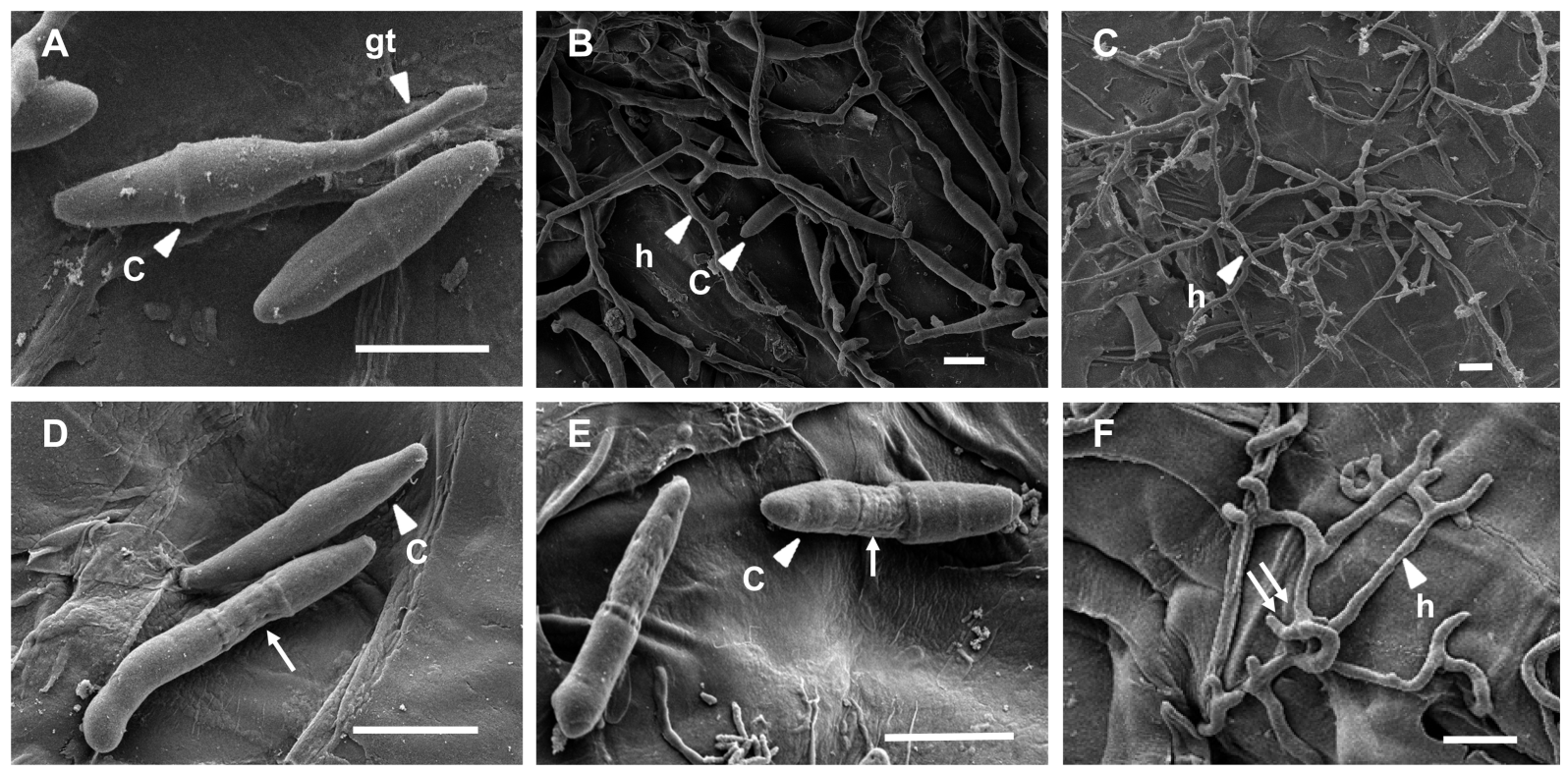

Fig. 6. Scanning electron microscopic observation of infection structures at 1, 2, and 3 days after inoculation on peels of kiwifruits untreated (A-C) and fungicide pyraclostrobin-boscalid (D-F). The concentration of fungicide and conidia suspension of Botryosphaeria dothidea were field application and $1 \times 10^{5}$ conidia/ml. Morphological change was observed on conidium (D and E, arrow) and branched hyphae (F, double arrows). Scale bars $=10 \mu \mathrm{m}$. c, conidium; h, hyphae; gt, gum tube.

germinated. At 3 days after inoculation some hyphae were observed which were frequently branched (Fig. $6 \mathrm{~F}$, double arrows), indicating the fungicide may suppress the hyphal growth.

\section{Discussion}

Although ripe rot caused by $B$. dothidea has been known in kiwifruit $A$. chinensis cultivars 'Haegum' and 'Hayward', there was no report announcing ripe rot on the cultivar 'Zesy002'. Over the last decade the postharvest disease has often occurred on 'Zesy002'; this cultivar is known to be tolerant to Psa (Zespri International Limited, 2016). In a previous study, $B$. dothidea was isolated from symptomatic kiwifruit and confirmed as the causal fungus of ripe rot by fulfilling Koch's Postulates (Ledesma et al., 2021).

Protection efficacy of agrochemicals against ripe rot in cultivar 'Hort16A' and 'Hayward' has been previously reported. Among three agrochemicals tested, benomyl WP showed the highest efficacy, followed by thiophanatemethyl WP and carbendazim + diethofencarb WP when they were treated in the orchards Jeju Island, Korea (Kim et al., 2013).

In this study, among the agrochemicals tested boscalid + fludioxonil was shown to have the most effective antifungal activity in the artificial medium (Figs. 1 and 2). The efficacy of this chemical was so high that its concentration had to dilute as 100 fold in the in vitro experiment. However, in the bio-test on fruit, this mixture was not the most effective among the tested fungicides. Unexpectedly, pyraclostrobin + boscalid mixture was the most effective against ripe rot on 'Zesy002' fruit (Fig. 4). It is not clearly explained why the efficacy of the agrochemicals was different between the in vitro and in vivo experiments. One of the reasons for this difference in efficacy could be that different inoculum types were used in each test (mycelium in the in vitro test and conidia in the in vivo test). It was also suggested that some factors such as fruit exudates or $\mathrm{pH}$ may influence the activity of the agrochemicals. (Mihaylova-Kroumova et al., 2020; Zhao et al., 2016).

Boscalid is a pyridinecarboxamide which is active against a broad range of fungal pathogens including Botrytis cinerea causing grey mold (Veloukas and Karaoglanidis, 2012) or Sclerotinia sclerotiorum causing stem rot in canola (Munoz, 2016). Boscalid has been known as an inhibitor of succinate dehydrogenase which has a role in electron transport chain in mitochondria (Hatefi, 1985). Also, pyraclostrobin is a carbamate ester which is widely used as a fungicide for control of many plant pathogens such as Alternaria spp. (Surviliene and Dambrauskiene, 2006) or Ascochyta rabiei (Chang et al., 2007). This agrochemical inhibits energy transfer in mitochondria in the fugal cell (Sierotzki and Scalliet, 2013). Mixtures with pyraclostrobin and boscalid were effective against brown 
spot on the leaves of pear in orchards (Petré et al., 2015).

The infection behavior of $B$. dothidea in kiwifruit is not well known yet. In this study, it was observed that the conidia of $B$. dothidea germinate fast similar to some other fungal pathogens. Also, it seems the fungus can invade the host cells without forming appressoria (Fig. 5). In order to illustrate how the agrochemicals inhibit fungal infection, the infection structures were observed on the surface of kiwifruit 'Zesy002' treated with pyraclostrobin + boscalid. On the agrochemical-treated kiwifruit there was a very low germination rate and restricted hyphal growth of $B$. dothidea (Table 2, Fig. 5). There have been previous reports that germination rates and hyphal development are decreased by this fungicide. Both germination rate and hyphal growth of Alternaria alternata and Penicillium digitatum were inhibited by boscalid treatment (Vega and Dewdney, 2015; $\mathrm{Xu}$ et al., 2020). Also, it was reported that the germination rate of Puccinia striiformis causing stripe rust in wheat (Kang et al., 2019) or Colletotrichum acutatum causing anthracnose in chili (Gao et al., 2017), was decreased by pyraclostrobin. Conidia and hyphae of Monilinia fructicola and Botrytis cinerea treated with mixtures of pyraclostrobin and boscalid did not germinate or develop well compared with untreated conidia and hyphae (Amiri et al., 2010; Kim and Xiao, 2010).

Electrion microscopy of the fine structure of $B$. dothidea on the untreated kiwifruit showed the intact conidia and hyphae (Fig. 6A-C); this was similar to those observed with the fluorescence microscope (Fig. 5). Strangely, the fungal samples for SEM grew more slowly than those used for fluorescence microscopy; this was likely to be due to environmental differences during the incubation period such as temperature. Through the observation with SEM it was clearly shown that mixtures of pyraclostrobin and boscalid may suppress the growth of $B$. dothidea resulting in the morphological change of conidia and branch of hyphae. Similar observations were found on the kiwifruit pretreated with other fungicides such as iminoctadine-tris or fluazinam (data not shown).

Therefore, it was suggested that the function of the agrochemicals in the suppression of ripe rot on kiwifruit may be the decreasing of germination rate and hyphal development of $B$. dothidea. However, for applying of agrochemicals to the fields, it would be necessary to undertake more experiments in order to prove their efficacy.

\section{Conflicts of Interest}

This work was financially supported by Zespri Group Limited, New Zealand.

\section{Acknowledgments}

This work was supported by Zespri Group Limited, New Zealand through a research and development service program and a research grant of Jeju National University in 2021.

\section{References}

Amiri, A., Brannen, P. M. and Schnabel, G. 2010. Reduced sensitivity in Monilinia fructicola field isolates from South Carolina and Georgia to respiration inhibitor fungicides. Plant Dis. 94:737-743.

Chang, K. F., Ahmed, H. U., Hwang, S. F., Gossen, B. D., Strelkov, S. E., Blade, S. F. and Turnbull, G. D. 2007. Sensitivity of field populations of Ascochyta rabiei to chlorothalonil, mancozeb and pyraclostrobin fungicides and effect of strobilurin fungicides on the progress of ascochyta blight of chickpea. Can. J. Plant Sci. 87:937-944.

Donati, I., Buriani, G., Cellini, A., Mauri, S., Costa, G. and Spinelli, F. 2014. New insights on the bacterial canker of kiwifruit (Pseudomonas syringae pv. actinidiae). J. Berry Res. 4:53-67.

Gao, Y.-Y., He, L.-F., Li, B.-X., Mu, W., Lin, J. and Liu, F. 2017. Sensitivity of Colletotrichum acutatum to six fungicides and reduction in incidence and severity of chili anthracnose using pyraclostrobin. Australas. Plant Pathol. 46:521-528.

Greer, G. and Saunders, C. 2012. The costs of Psa-V to the New Zealand kiwifruit industry and the wider community: report to kiwifruit vinehealth. Lincoln University, Christchurch, New Zealand. 75 pp.

Hatefi, Y. 1985. The mitochondrial electron transport and oxidative phosphorylation system. Annu. Rev. Biochem. 54:10151069.

Kang, Z., Li, X., Wan, A., Wang, M. and Chen, X. 2019. Differential sensitivity among Puccinia striiformis f. sp. tritici isolates to propiconazole and pyraclostrobin fungicides. Can. J. Plant Pathol. 41:415-434.

Kim, G. H., Jung, J. S. and Koh, Y. J. 2017a. Occurrence and epidemics of bacterial canker of kiwifruit in Korea. Plant Pathol. J. 33:351-361.

Kim, G. H. and Koh, Y. J. 2018. Incidence rates of major diseases on green-fleshed kiwifruit cv. hayward and yellow-fleshed kiwifruit cv. haegeum. Res. Plant Dis. 24:175-181.

Kim, G. H., Lee, Y. S., Jung, J. S., Hur, J.-S. and Koh, Y. J. 2013. Optimal spray time, interval and number of preventive fungicides for the control of fruit rots of green and gold kiwifruit cultivars. Res. Plant Dis. 19:1-6.

Kim, M.-J., Kwon, Y., Shin, M. H., Kim, J. G., Kwak, Y.-S. and Kwack, Y.-B. 2017b. Fungal infection occurrence on new cultivars of kiwifruit correlated with fruit soluble solids content and firmness. Korean J. Soil Sci. Fertil. 50:215-225.

Kim, Y. K. and Xiao, C. L. 2010. Resistance to pyraclostrobin and boscalid in populations of Botrytis cinerea from stored 
apples in Washington State. Plant Dis. 94:604-612.

Koh, Y. J., Jung, J. S. and Hur, J. S. 2003. Current status of occurrence of major diseases on kiwifruits and their control in Korea. Acta Hortic. 610:437-443.

Ledesma, M., Shin, Y. H., Whiteman, S., Tyson, J., Kim, G. H., Hong, J. K., Zange, B. and Jeun, Y. C. 2021. Causal fungus of side rot on 'Zesy002' kiwifruits in Jeju Island, South Korea. Res. Plant Dis. 27:24-31.

Manning, M. A., Meier, X., Olsen, T. L. and Johnston, P. R. 2003. Fungi associated with fruit rots of Actinidia chinensis 'Hort16A' in New Zealand. N. Z. J. Crop Hortic. Sci. 31:315-324.

Mihaylova-Kroumova, A. B., Artiouchine, I., Korenkov, V. D. and Wagner, G. J. 2020. Patterns of inheritance of acylsugar acyl groups in selected interspecific hybrids of genus Nicotiana. J. Plant Res. 133:509-523.

Munoz, C. L. 2016. Sensitivity of Sclerotinia sclerotiorum isolates from North Central us to azoxystrobin and boscalid. M.S. thesis. North Dakota State University, Fargo, ND, USA.

Petré, R., Labourdette, G., Braun, C. A., Meredith, R., Hauke, K., Van Hemelrijck, W., Schoofs, H., Deckers, T., Keulemans, W., Schoevaerts, C., Becker, R. C. and De Maeyer, L. 2015. Fosetyl-al (Aliette ${ }^{\circledR}$ ), a plant defense enhancer with good efficacy on bacteria and on ascomycetes in apples and pears. Acta Hortic. 1094:431-438.

Sierotzki, H. and Scalliet, G. 2013. A review of current knowledge of resistance aspects for the next-generation succinate dehydrogenase inhibitor fungicides. Phytopathology 103:880-
887.

Singletary, K. 2012. Kiwifruit: overview of potential health benefits. Nutr. Today 47:133-147.

Survilienè, E. and Dambrauskienè, E. 2006. Effect of different active ingredients of fungicides on Alternaria spp. growth in vitro. Agron. Res. 4:403-406.

Tridge. 2021. South Korea's kiwifruit export, export trends, and top export destinations. URL https://www.tridge.com/ko/intelligences/kiwifruit/export [7 September 2020].

Vega, B. and Dewdney, M. M. 2015. Sensitivity of Alternaria alternata from citrus to boscalid and polymorphism in ironsulfur and in anchored membrane subunits of succinate dehydrogenase. Plant Dis. 99:231-239.

Veloukas, T. and Karaoglanidis, G. S. 2012. Biological activity of the succinate dehydrogenase inhibitor fluopyram against Botrytis cinerea and fungal baseline sensitivity. Pest Manag. Sci. 68:858-864.

Xu, Q., Zhang, K., Fu, Y., Ma, H. and Zhu, F. 2020. Toxic action and baseline sensitivity of boscalid against Penicillium digitatum. Crop Prot. 137:105272.

Zespri International Limited. 2016. Zespri annual review 2015/16. Zespri International Limited., Mount Maunganui, New Zealand. 22 pp.

Zhao, L., Huang, Y., Hu, J., Zhou, H., Adeleye, A. S. and Keller, A. A. 2016. ${ }^{1} \mathrm{H}$ NMR and GC-MS based metabolomics reveal defense and detoxification mechanism of cucumber plant under nano-cu stress. Environ. Sci. Technol. 50:2000-2010. 